\title{
ADVANCED SURFACE-REFLECTED RADIANCE CORRECTION FOR AIRBORNE HYPERSPECTRAL IMAGERY IN COASTAL RED TIDE DETECTION
}

\author{
Jee-Eun Min ${ }^{1,4 *}$, Seung-Kuk Lee ${ }^{2}$ Joo-Hyung Ryu ${ }^{3}$ \\ ${ }^{1}$ Institute of Environmental Geoscience, Pukyong National University, Busan, Republic of Korea \\ ${ }^{2}$ Department of Earth \& Environmental Science, Pukyong National University, Busan, Republic of Korea \\ ${ }^{3}$ Korea Ocean Satellite Center, Korea Institute of Ocean Science \& Technology, Busan, Republic of Korea \\ ${ }^{4}$ Underwater Survey Technology 21, Incheon, Republic of Korea
}

KEY WORDS: Hyperspectral image, Atmospheric correction, Surface-reflected radiance, Red tide

\begin{abstract}
:
Red tides are among the most common coastal hazards, causing serious damage to the coastal environment. Many satellite sensors can detect red tide blooms, but are limited in their detection of the exact area of the bloom and biological abundance in terms of spatial and spectral resolution. The high spatial and spectral resolutions of hyperspectral airborne remote sensing data may help overcome these limitations to analyze red tide blooms more effectively. To identify potential applications of hyperspectral airborne data in red tide detection, an integrated field campaign was performed in September 2016 off the coast of Tongyeong, South Korea. An AisaEAGLE sensor was installed on a Cessna 208B crewed aircraft to obtain hyperspectral images of an $18 \mathrm{~km} \times 18 \mathrm{~km}$ coastal area. To assess the atmospheric correction of the hyperspectral data, in situ optical data and water samples were measured on two vessels concurrent with the flight path. Advanced surface-reflected radiance $\left(L_{\mathrm{r}}\right)$ correction and basic atmospheric path radiance $\left(L_{\mathrm{a}}\right)$ correction were performed on the hyperspectral images. Of these, $L_{\mathrm{r}}$ correction comprised a large proportion of the atmospheric correction. The atmosphere-corrected remote sensing reflectance data of the hyperspectral images closely matched the in-situ measurements. The data were assessed for red tide events using ratio analysis and the fluorescence line height technique; the ratio analysis more effectively detected regions with suspected red tides.
\end{abstract}

\section{INTRODUCTION}

Red tides, a common name for harmful algal bloom, are among the most common coastal disasters, causing serious damage to the coastal marine environment in South Korea (Kim et al., 2004; Lee et al., 2013). Although many satellite sensors are capable of detecting red tides, they are limited in their abilities to accurately analyze the exact shape and area of the red tide patch and the biological abundance in terms of spatial and spectral resolution. Aircraft-mounted hyperspectral sensors may overcome these limitations because of their high spatial and spectral resolutions, while remaining more cost-effective.

Imaging spectrometry (i.e., hyperspectral imaging) began at the Jet Propulsion Laboratory of the National Aeronautics and Space Administration (NASA) in the early 1980s, and enabled the acquisition of images on continuous spectrum channels. This method provides complete reflectivity spectrum information within a wavelength region for each pixel within an area (Gao et al., 2009). Hyperspectral remote sensing, which provides information regarding the overall spectral spectrum of the object, has been used for terrestrial studies in various fields (e.g., geology, minerology, agriculture, and forestry). In recent decades, it has also been applied to coastal and inland water quality research (Thiemann and Kaufmann, 2002; Brando and Dekker, 2003; Karaska et al., 2004; Vahtmae et al., 2006; Giardino et al., 2007; Ryan et al., 2009; Olmanson et al., 2013; Guild et al., 2020; Shin et al., 2020). However, it is difficult to use airborne hyperspectral imaging data for coastal environment analysis. Due to the comparatively large field of view (FOV) and complex pixel-based geometry, various pre-processing steps are required, and data verification is important. Due to these difficulties, a simple atmospheric correction based on radiative transfer model or provided on SW was used for the coastal

*minjeblue@gmail.com / jemin@ust21.co.kr ecosystem monitoring using aerial remote sensing data (Guild et al., 2020; Shin et al., 2020). In addition, for some data obtained at low altitudes, atmospheric correction was not performed (Guild et al., 2020). In this study, compared with the atmospheric correction using existing radiative transfer models, the advanced atmospheric correction was applied by correcting the angular reflected radiation on the sea surface to enable effective detection of red tide blooms using hyperspectral datasets.

\section{METHODOLOGY}

\subsection{Study area and data acquisition}

The study was performed in Tongyeong, South Korea, which is known for its high occurrence of red tides in summer. It also hosts the largest fish farms in South Korea, and experiences the greatest damage from red tide blooms (Lee et al., 2013). The field campaign for the acquisition and verification of hyperspectral

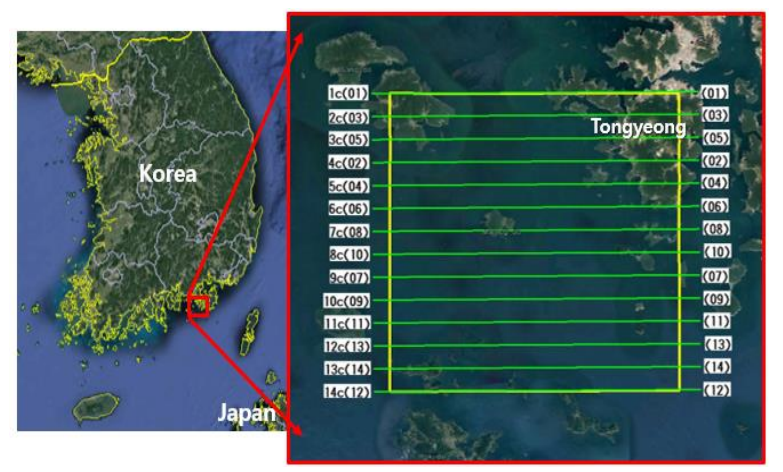

Figure 1. Study area of the coastal area around Tongyeong, South Korea and the planned flight lines for hyperspectral imaging. 


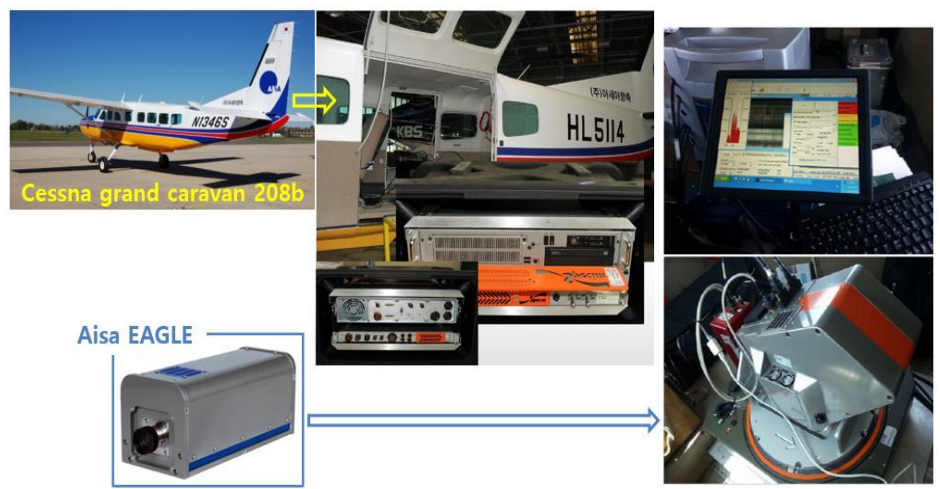

Figure 2. AisaEAGLE sensor mounted on the Cessna 208b aircraft and internal equipment installation.

images was conducted on September 24, 2016, in the coastal area of Tongyeong. Figure 1 shows the location of the study area and the planned aircraft flight path. Hyperspectral images were acquired with an AisaEAGLE hyperspectral sensor (Specim, Spectral Imaging Ltd., Oulu, Finland) mounted on a crewed Cessna 208B (Cessna, Wichita, KS, USA) operated by ASIA Aero Survey Co., Ltd. (Seoul, South Korea) (Figure 2). Table 1 lists detailed information regarding the aircraft and sensor. Because AisaEAGLE is a push-broom scanning linear sensor, 10 line-scan images with $30 \%$ sidelap were obtained in the $18 \mathrm{~km} \times$ $18 \mathrm{~km}$ study area (Figure 3). The spatial resolution was $2.0 \mathrm{~m}$, because of the flight height of $3,000 \mathrm{~km}$, and approximately 130 bands of spectral data were measured within $400-1000 \mathrm{~nm}$.

To verify the obtained hyperspectral images, match-up data were measured on two vessels at the same time and location as the corresponding flight route scheduled from 09:00 to 12:00 on September 24, 2016. In total, eight match-up datasets were obtained for the hyperspectral data verification. Water and sky radiance on the sea surface were measured using a spectroradiometer (ASD FieldSpec-3; Malvern Panalytical, Malvern, UK) to verify the result of atmospheric correction (Figure 4a). The marine environmental information included the absorption coefficients of sea water components for chlorophyll, suspended particulate matter (SPM), and dissolved organic matter $(\mathrm{DOM})$, as well as the concentrations of chlorophyll $\left(C_{\mathrm{ph}}\right)$ and SPM ( $C_{\text {SPM }}$ ) (Figure $\left.4 b\right)$. A spectrophotometer (Cary 100; Agilent, Santa Clara, CA, USA) was used to measure the absorption coefficients of total particles ( $\left.a_{\text {total }}\right)$, SPM ( $a$ SPM $)$, and $\operatorname{DOM}\left(a_{\text {DOM }}\right)$. The absorption coefficient of chlorophyll $\left(a_{\mathrm{ph}}\right)$ was calculated by subtracting $a_{\text {SPM }}$ from $a_{\text {total }} . C_{\text {ph }}$ was calculated using the trichroic equation (Jeffrey and Humphrey, 1975) and

\begin{tabular}{|c|c|}
\hline \multicolumn{2}{|c|}{ Cessna 208B } \\
\hline Size $(\mathrm{W} \times \mathrm{L} \times \mathrm{H})$ & $15.9 \mathrm{~m} \times 12.7 \mathrm{~m} \times 4.7 \mathrm{~m}$ \\
\hline Weight $($ empty $)$ & $2,067 \mathrm{~kg}$ \\
\hline Maximum weight & $3,969 \mathrm{~kg}$ \\
\hline \multirow{2}{*}{ Operation Speed } & Minimum $120 \mathrm{kn}(222 \mathrm{~km} / \mathrm{h})$ \\
\cline { 2 - 2 } & Maximum $185 \mathrm{kn}(343 \mathrm{~km} / \mathrm{h})$ \\
\hline Flying Range & Maximum $1,996 \mathrm{~km}$ \\
\hline Flying Height & Maximum 7,620 m \\
\hline \multicolumn{2}{|c|}{ AisaEAGLE } \\
\hline Spectral range & $400 \sim 1,000 \mathrm{~nm}$ \\
\hline Spectral band & Maximum 488 bands \\
\hline Spatial pixels & 1,024 pixels \\
\hline Swath width & $2,048 \mathrm{~m}$ (at a height of 3,000 m) \\
\hline Ground Resolution & $2 \mathrm{~m}$ (at a height of 3,000 m) \\
\hline Data interface & 12 bits Cameralink \\
\hline
\end{tabular}

Table 1. Information regarding the Cessna 208B aircraft and AisaEAGLE hyperspectral sensor.
$C_{\text {SPM }}$ was measured gravimetrically after filtration on pre-dried $\left(60^{\circ} \mathrm{C}, 4 \mathrm{~h}\right) 47-\mathrm{mm}$ polycarbonate membrane filters. All data acquisition methods and calculations were performed in accordance with the NASA Ocean Optics Protocols (Mueller et al., 2003) and International Ocean Colour Coordinating Group (IOCCG) Protocol Series (Boss et al., 2018).

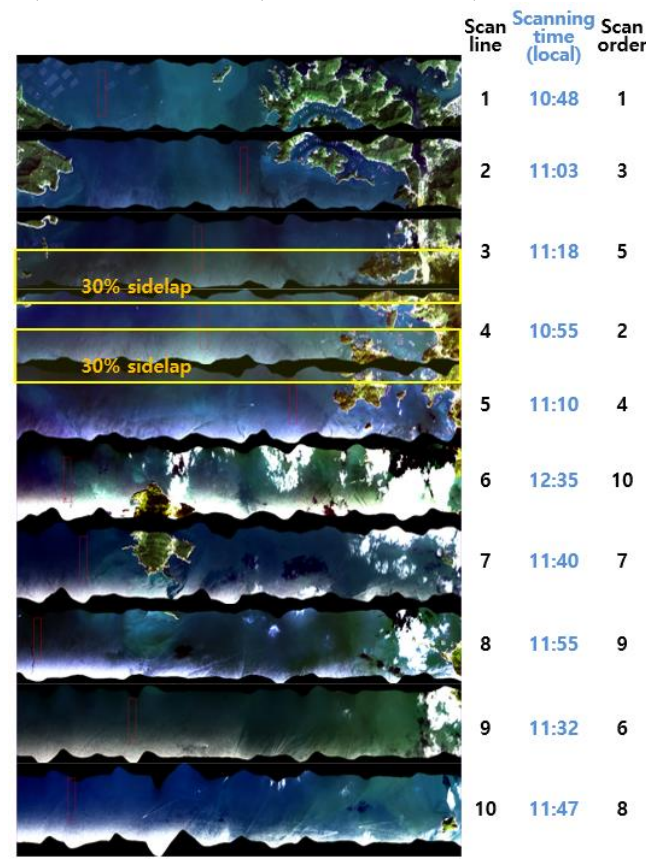

Figure 3. Hyperspectral scanning images for each scan line and scanning order and time information for capturing images.
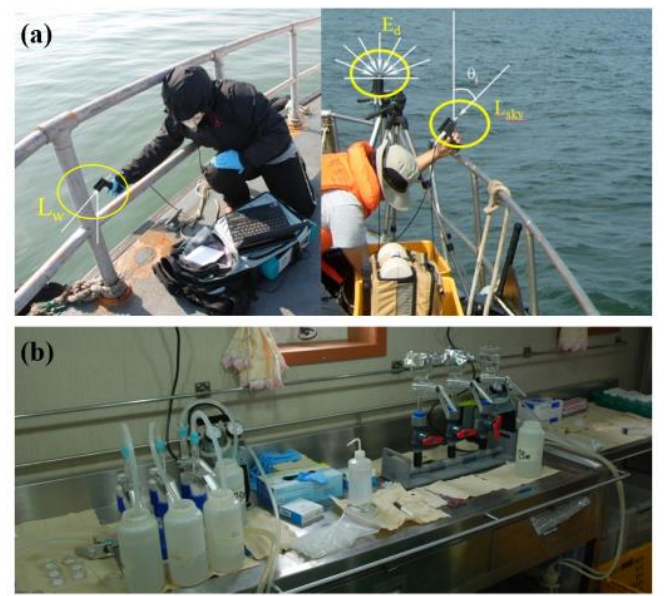

Figure 4. In-situ field data measurements for (a) sea surface radiance and (b) environmental characteristics. 


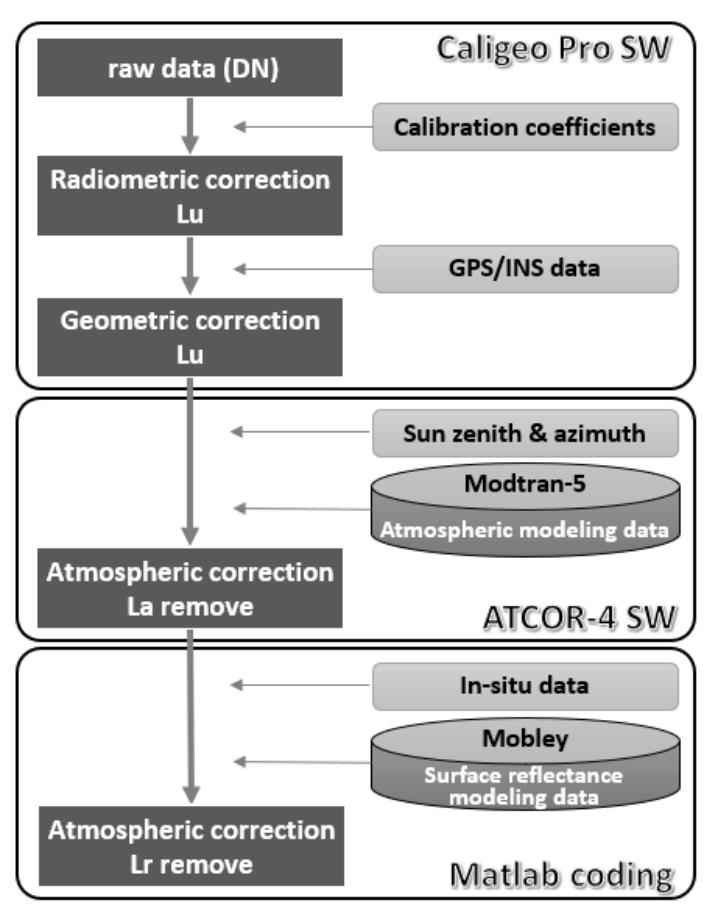

Figure 5. Flowchart of the data processing procedures used on the hyperspectral scanning data.

\subsection{Hyperspectral data pre-processing}

The raw data (digital number) captured by the hyperspectral camera were processed sequentially for radiometric correction, georeferencing, atmospheric correction, and mosaic processing. Figure 5 presents the data processing procedure. First, radiometric and geometric corrections were performed using CaligeoPRO software (Specim, Spectral Imaging Ltd.). Radiometric correction was initially accomplished to compensate for the scattering of radiation due to atmospheric scattering, the geometrical relationship between target and sensor, and the sensor response characteristics. Then, geometric correction was performed using the Global Positioning System/Inertial Navigation System (GPS/INS) and calibration dataset. In this process, the coordinate information of each pixel was obtained by synchronizing the image with the GPS/INS data to generate an orthorectified image.
Atmospheric correction was performed in two steps. Generally, an aircraft-mounted sensor viewing the ocean measures upwelling radiance that includes contributions by the atmosphere, water surface, and water column (Mobley et al. 2020), as illustrated in Figure 6. Atmospheric path radiance $\left(L_{\mathrm{a}}\right)$ is the atmospheric scattering effect caused by solar radiance scattered one or more times by atmospheric gases and aerosols into the direction of the sensor. Surface-reflected radiance (i.e., sun and sky glint; $L_{\mathrm{r}}$ ) represents downwelling solar radiance reflected toward the sensor off the water surface. When the light source is transmitted through the surface in the ocean $\left(L_{t}\right)$, it is altered by absorbing and scattering components in the water. $L_{\mathrm{t}}$ is then scattered upward and transmitted back through surface toward the sensor as water-leaving radiance $\left(L_{\mathrm{w}}\right)$. Total radiance $\left(L_{\mathrm{u}}\right)$ represents the sensor scanning data, including the atmospheric contributions of $L_{\mathrm{a}}$ and $L_{\mathrm{r}}$, as well as $L_{\mathrm{w}}$ (the value of interest in this study). To obtain $L_{\mathrm{w}}, L_{\mathrm{a}}$ and $L_{\mathrm{r}}$ were subtracted from $L_{\mathrm{u}}$ (Eq. 1). In this study, $L_{\mathrm{a}}$ was corrected using the Atmospheric and Topographic CORrection (ATCOR) software (ReSe Applications, Wil, Switzerland), based on MODTRAN5 modeling. $L_{\mathrm{r}}$ was corrected using in situ sky radiance $\left(L_{\mathrm{sky}}\right)$ measurements and the surface reflection model proposed by Mobley et al. (2015).

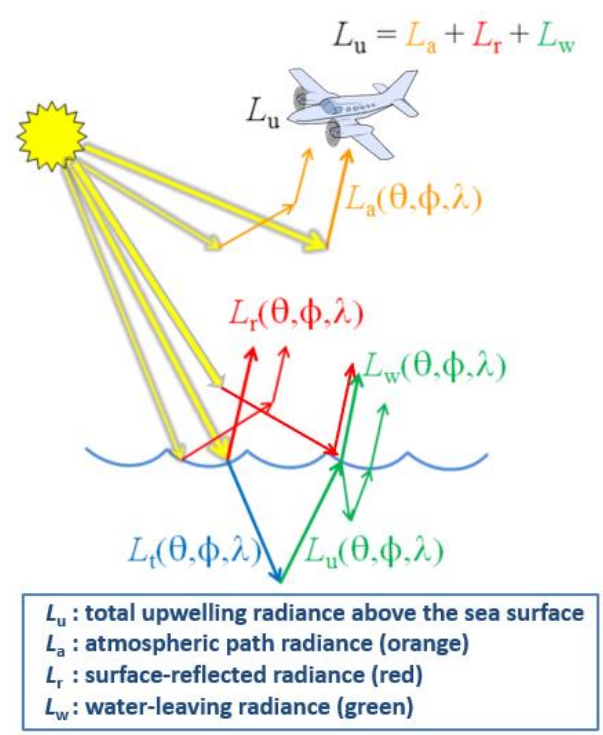

Figure 6. Conceptual diagram of the contributions to the total upwelling radiance above the sea surface $\left(L_{\mathrm{u}}\right)$

(Mobley et al., 2020).

\begin{tabular}{|c|c|c|c|c|c|c|c|c|c|c|c|c|c|c|c|c|c|}
\hline \multirow{2}{*}{$\begin{array}{c}\text { Scan } \\
\text { Line } \\
\#\end{array}$} & \multirow{2}{*}{$\begin{array}{c}\text { Scan } \\
\text { time } \\
\text { local } \\
\text { (hh:mm) }\end{array}$} & \multirow{2}{*}{$\begin{array}{l}\text { Scan } \\
\text { order }\end{array}$} & \multicolumn{2}{|c|}{$\begin{array}{c}\text { Zenith } \\
\left(-18.85^{\circ} \sim\right. \\
\left.18.89^{\circ}\right)\end{array}$} & \multirow{2}{*}{$\begin{array}{l}\text { Flight } \\
\text { direction }\end{array}$} & \multicolumn{2}{|c|}{ Azimuth $\left({ }^{\circ}\right)$} & \multicolumn{2}{|c|}{ Solar geometry $\left({ }^{\circ}\right)$} & \multicolumn{2}{|c|}{$\varphi_{v}\left({ }^{0}\right)$} & \multicolumn{6}{|c|}{$\begin{array}{l}\text { Surface reflectance factors }(\rho) \\
\text { by Mobley's model (Figure } 8 \text { ) }\end{array}$} \\
\hline & & & Upper & Lower & & Upper & Lower & Azimuth & Elevation & Upper & Lower & case & $-20^{\circ}$ & $-10^{\circ}$ & $0^{\circ}$ & $10^{\circ}$ & $20^{\circ}$ \\
\hline 1 & $10: 40$ & 1 & + & - & to West & 4.2 & 184.2 & 141.58 & 47.82 & 222.62 & 42.62 & (4) & 0.12 & 0.12 & 0.09 & 0.06 & 0.04 \\
\hline 2 & $10: 55$ & 3 & + & - & to West & 301.6 & 121.6 & 146.56 & 49.62 & 155.04 & -24.96 & (11) & 0.14 & 0.14 & 0.09 & 0.06 & 0.04 \\
\hline 3 & $11: 10$ & 5 & + & - & to West & 1.8 & 181.8 & 151.91 & 51.20 & 209.89 & 29.89 & (3) & 0.14 & 0.14 & 0.09 & 0.06 & 0.04 \\
\hline 4 & $10: 48$ & 2 & - & + & to East & 357.7 & 177.7 & 144.19 & 48.81 & 213.51 & 33.51 & (3) & 0.14 & 0.14 & 0.09 & 0.06 & 0.04 \\
\hline 5 & 11:03 & 4 & - & + & to East & 357.9 & 177.9 & 149.37 & 50.49 & 208.53 & 28.53 & (3) & 0.14 & 0.14 & 0.09 & 0.06 & 0.04 \\
\hline 6 & $12: 27$ & 10 & + & - & to West & 1.9 & 181.9 & 183.60 & 54.60 & 178.3 & -1.7 & (1) & 0.2 & 0.2 & 0.09 & 0.05 & 0.04 \\
\hline 7 & $11: 32$ & 7 & - & + & to East & 358.2 & 178.2 & 160.36 & 53.02 & 197.84 & 17.84 & (2) & 0.2 & 0.2 & 0.09 & 0.05 & 0.04 \\
\hline 8 & 11:47 & 9 & - & + & to East & 358.1 & 178.1 & 166.49 & 53.90 & 191.61 & 11.61 & (2) & 0.2 & 0.2 & \begin{tabular}{|l|}
0.09 \\
\end{tabular} & 0.05 & 0.04 \\
\hline 9 & $11: 26$ & 6 & + & - & to West & 1.8 & 181.8 & 157.99 & 52.58 & 203.81 & 23.81 & (3) & 0.14 & 0.14 & \begin{tabular}{|l|l|}
0.09 \\
\end{tabular} & \begin{tabular}{|l|l|}
0.06 \\
\end{tabular} & 0.04 \\
\hline 10 & $11: 40$ & 8 & + & - & to West & 1.8 & 181.8 & 163.60 & 53.53 & 198.2 & 18.2 & (2) & 0.2 & 0.2 & \begin{tabular}{|l|l|}
0.09 \\
\end{tabular} & 0.05 & 0.04 \\
\hline
\end{tabular}

Table 2. Information regarding hyperspectral image acquisition. 
ATCOR software provides atmospheric and topographic corrections to eliminate atmospheric and topographic effects and derive physical values from the Earth's surface, such as surface reflectivity spectra, radiation rates, and surface temperatures (Richter and Schläpfer, 2017). There are three ATCOR models available, two for satellite imagery (ATCOR-2 and -3 ) and one for airborne imagery (ATCOR-4), where the numbers 2-4 represent the geometric degrees of freedom (DOF). ATCOR-2 and -3 were developed for satellite imagery of flat terrain $(2$ geometric DOF) and mountainous terrain (3 geometric DOF), respectively. By contrast, ATCOR-4 includes 4 geometric DOF for $\mathrm{x}, \mathrm{y}, \mathrm{z}$, and scan angle, among which scan angle is important for correcting atmospheric effects of airborne images because most airborne sensors have a large FOV.

Input image and scan angle files are required for ATCOR processing. The scan angle file was generated with CaligeoPRO software (Figure 5) using the "ATCOR4f: flat terrain" mode to represent marine data. Next, the sensor type, flight operation information, and solar geometry values were set for sensor and calibration file generation (detailed information is listed in Table 2 ), and the 'maritime' aerosol type was selected. Finally, $L_{\mathrm{a}}$ corrected data from total radiance were obtained.

\subsection{Correction of Surface-reflected radiance $\left(L_{r}\right)$}

$L_{\mathrm{u}}$ is a function of viewing direction $(\theta)$, azimuth angle $(\varphi)$, and wavelength $(\lambda)$; it encompasses $L_{\mathrm{a}}$ and $L_{\mathrm{r}}$, as well as $L_{\mathrm{w}}$ (the parameter of interest in this study), as shown in Eq. (1). $L_{\mathrm{a}}$ was easily removed using ATCOR. However, $L_{\mathrm{r}}$ consists of three components (Figure 7): direct solar reflection (i.e., sun glint; $L_{\mathrm{r}}$ (1), Figure 7), sun and sky reflection values from adjacent pixels $\left(L_{\mathrm{r}}-(2)\right.$, Figure 7), and sky-reflected radiance $\left(L_{\mathrm{r}}-(3)\right.$, Figure 7$)$.

Sun-glint is a mirror-like specular reflection of sunlight reflected off water back at the sensor, which occurs when sunlight reflects off the surface of water at an angle identical to the angle at which the satellite sensor views it. Sun glint $\left(L_{\mathrm{r}}\right.$-(1) was eliminated because it comprises a large proportion of $L_{\mathrm{r}}$. The white area at the bottom of each scan line represents the sun-glint region (Figure 3). Large sun-glint regions were observed in the images obtained in this study, which expanded as time approached noon and the sun's elevation increased. In this study, calibration was achieved by eliminating values $\geq 0.0035$ in the NIR band of $865 \mathrm{~nm}$. Sun and sky reflection from adjacent pixels $\left(L_{\mathrm{r}}\right.$-(2) $)$ is difficult to calibrate, and was thus not calibrated in this study. Finally, sky-reflected radiance $\left(L_{\mathrm{r}}-(3)\right)$ was corrected using in situ $L_{\text {sky }}$ data and a surface reflection model. $L_{\mathrm{r}}$ can be modelled as the product of surface radiance reflection $(\rho)$ multiplied by $L_{\text {sky }}$, as shown in Eq. (2).

$$
\begin{gathered}
L_{w}(\theta, \varphi, \lambda)=L_{u}(\theta, \varphi, \lambda)-L_{a}(\theta, \varphi, \lambda)-L_{r}(\theta, \varphi, \lambda) \\
L_{w}(\theta, \varphi, \lambda)=L_{u}(\theta, \varphi, \lambda)-L_{a}(\theta, \varphi, \lambda)-\rho\left(\theta, \varphi, \theta_{0}, W\right) L_{s k y}(\theta, \varphi, \lambda)
\end{gathered}
$$

where $\theta$ : viewing direction (zenith angle) for each pixel (same as $\theta_{v}$ in Mobley's model)

$\varphi$ : azimuth angle for each pixel from the North direction

$\theta_{0}$ : solar zenith angle

$\rho:$ radiance reflection

$\lambda$ : wavelength

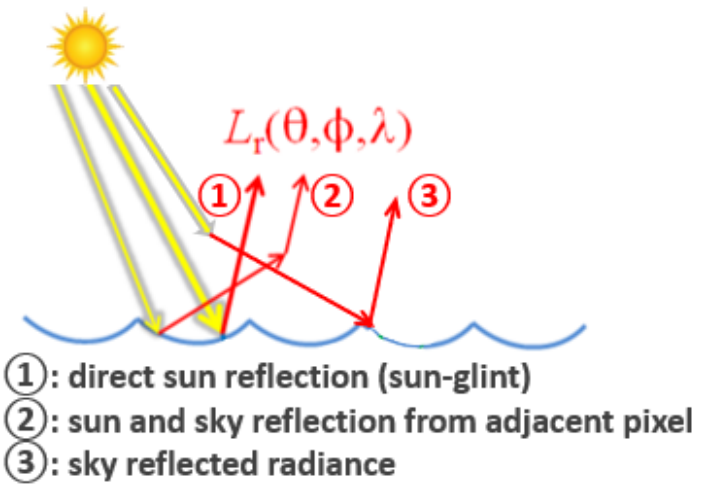

Figure 7. Conceptual diagram of the surface-reflected radiance $\left(L_{\mathrm{r}}\right)$.

Mobley (2015) generated $\rho$ at the sea surface as a function of viewing direction and azimuth angle of the sensor, solar zenith angle, and wind speed. Figure 8 shows the $\rho$ modelling result as a function of sensor viewing angle from nadir and sensor azimuth angle from the sun in the case of a fixed solar zenith angle $\left(\theta_{0}\right)$ of $40^{\circ}$ and wind speed of $10 \mathrm{~m} \mathrm{~s}^{-1}$. Because the sensor viewing angles for pixels were distributed between $-18.85^{\circ}$ and $18.89^{\circ}, \rho$ values within the red dotted line in Figure 8 were used for $L_{\mathrm{r}}$ correction. According to the relative azimuth angle from the sun to the sensor $\left(\varphi_{\mathrm{v}}\right)$ for each scan line, the $\rho$ values were extracted from Figure 8 and listed in Table 2. $L_{\mathrm{r}}$ was calculated using the extracted $\rho$ values (Table 2) and in situ $L_{\text {sky }}$ data. Both $L_{\mathrm{r}}$ and $L_{\mathrm{a}}$ were subtracted from $L_{\mathrm{u}}$ to obtain $L_{\mathrm{w}}$. Finally, remote sensing reflectance $\left(R_{\mathrm{rs}}\right)$ was calculated by dividing $L_{\mathrm{w}}$ into in situ downwelling radiance.

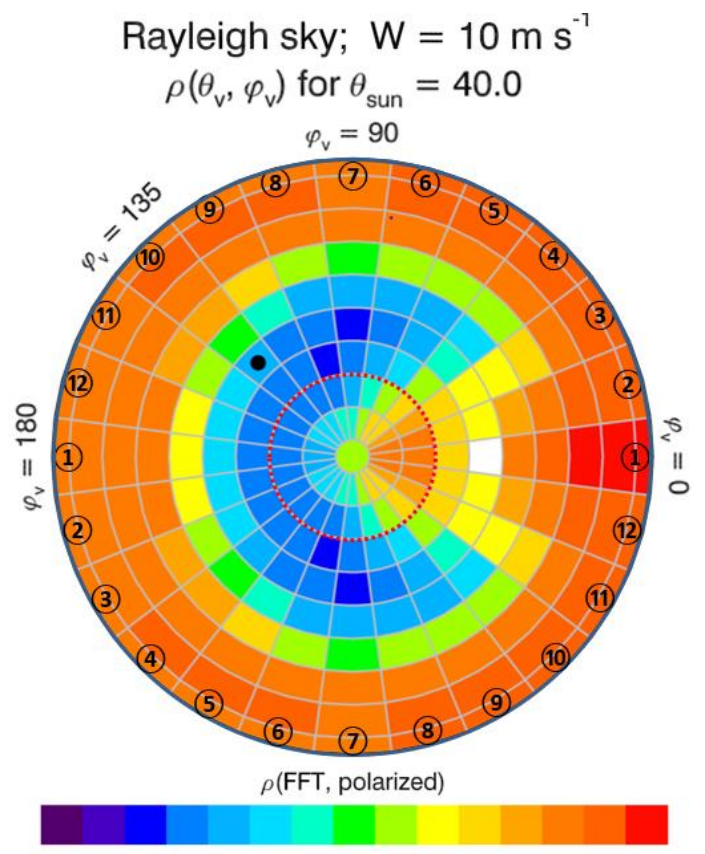

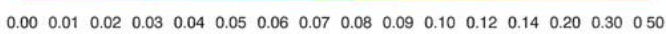

Figure 8. Surface radiance reflection $(\rho)$ as a function of offnadir and azimuthal viewing directions (relative to the sun's azimuth at $\varphi_{v}=0$ ) for a single sun zenith angle of $\theta_{0}=40^{\circ}$, computed using FFT surface and polarized ray tracing with a Rayleigh sky and a wind speed of $10 \mathrm{~m} \mathrm{~s}^{-1}$ (Mobley, 2015). 


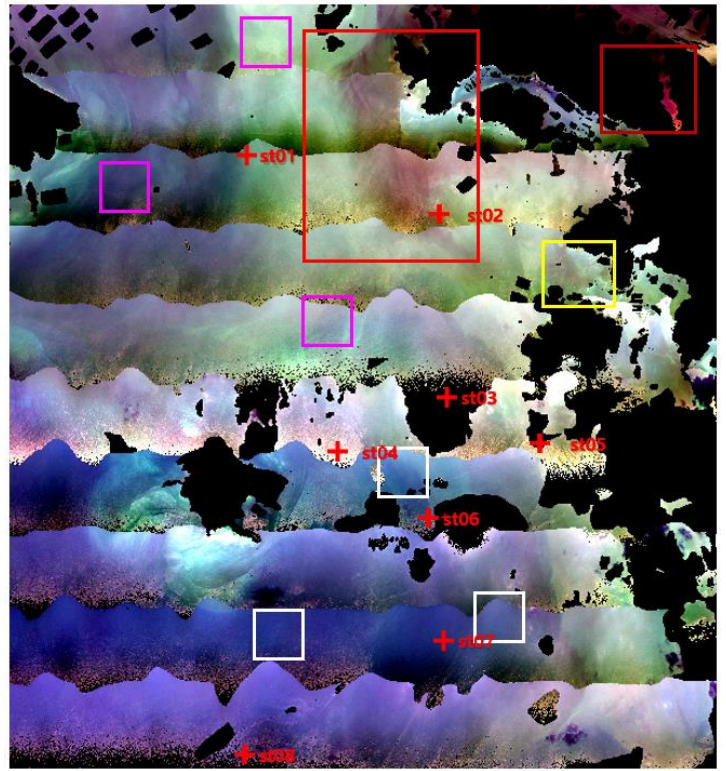

Figure 9. Natural color RGB composite for the mosaic image of atmospheric-corrected hyperspectral images

(red crosses: in-situ sampling sites for atmospheric correction verification).

\section{RESULTS}

\subsection{Verification of atmospheric correction results}

Figure 9 shows the mosaic $R_{\mathrm{rs}}$ image of atmosphere-corrected hyperspectral scanning data for path radiance and surface reflection. Compared with the uncorrected images (Figure 3), the sun-glint region and high-reflection regions were well corrected. The atmosphere-corrected results were verified using in situ $R_{\mathrm{rs}}$ data, which matched the airborne images. In total, eight sets of in situ data were acquired, but two datasets were not matched with the airborne data because of cloud masking. The sampling locations of the match-up data are illustrated by red crosses in Figure 9, and the obtained in situ $R_{\mathrm{rs}}$ spectrum is displayed as a green line in Figure 10. Stations st01 and st07 were located at the upper part of the line, which was comparatively unaffected by sun glint, whereas stations st02, st04, st06, and st 08 were located at the lower part of the line, which was comparatively strongly affected by sun glint. The in-situ $R_{\mathrm{rs}}$ spectrum was compared with
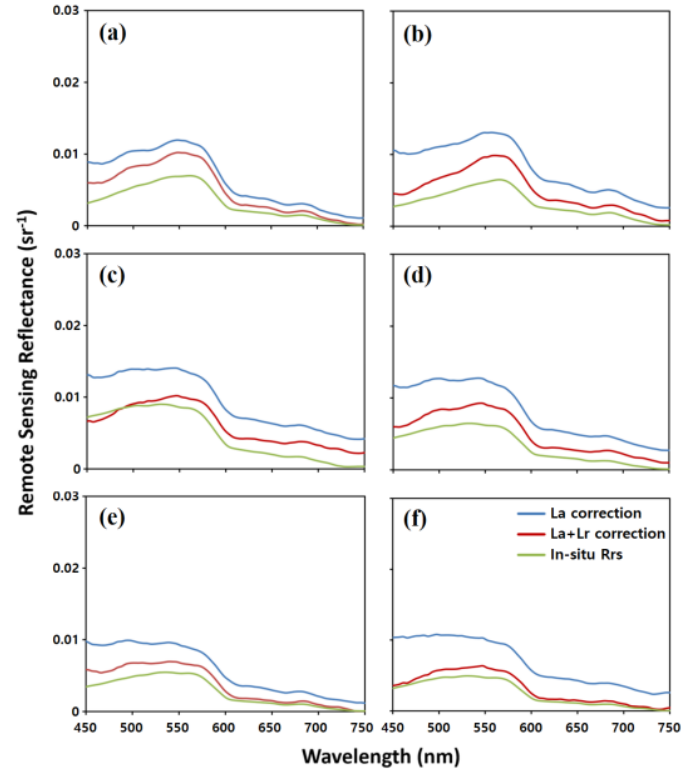

Figure 10. Comparison of the $R_{\mathrm{rs}}$ spectra of $L_{\mathrm{a}}$ correction (blue line), $L_{\mathrm{a}}$ and $L_{\mathrm{r}}$ correction (red line), and in-situ measurements (greed line) for sampling stations (a) st01, (b) st02, (c) st04, (d) st06, (e) st07, and (f) st08.

$L_{\mathrm{a}}$-corrected (blue line, Figure 10$)$ and $L_{\mathrm{a}}+L_{\mathrm{r}}$-corrected (red line, Figure 10) results. The $L_{\mathrm{a}}$-corrected $R_{\mathrm{rs}}$ spectrum showed values 2 3-fold higher than the in-situ dataset throughout the wavelength range. By contrast, the $L_{\mathrm{a}}+L_{\mathrm{r}}$-corrected $R_{\mathrm{rs}}$ spectrum had values similar to the in-situ $R_{\mathrm{rs}}$ data, and effectively eliminated atmospheric effects.

The shapes of the $L_{\mathrm{a}}$-corrected and $L_{\mathrm{a}}+L_{\mathrm{r}}$-corrected $R_{\mathrm{rs}}$ spectra were similar to the shape of the in situ $R_{\mathrm{rs}}$ spectrum throughout the wavelength range. Notably, $L_{\mathrm{a}}+L_{\mathrm{r}}$-corrected $R_{\mathrm{rs}}$ at st 08 was almost identical to the in situ $R_{\mathrm{rs}}$ data. However, there were small differences within the wavelength range of $450 \sim 550 \mathrm{~nm}$, especially at stations located at the upper part of the line (st01 and st07, Figure 10a and e). For stations located at the lower part of the line (st02, st04, st06, and st08, Figure 10b, c, d, and f), the $R_{\mathrm{rs}}$ values were eliminated more within $450 \sim 500 \mathrm{~nm}$ than at other wavelengths. With respect to st04, located within the area most affected by sun glint, the atmospheric effect was overcalibrated within $450 \sim 500 \mathrm{~nm}$, with $R_{\mathrm{rs}}$ values smaller than values in the in-situ $R_{\mathrm{rs}}$ data. This result was due to excessive correction of $L_{\mathrm{r}}$, rather than $L_{\mathrm{a}}$. By contrast, within $650 \sim 750 \mathrm{~nm}$, less $L_{\mathrm{r}}$ correction was applied than at other wavelengths. Overall,
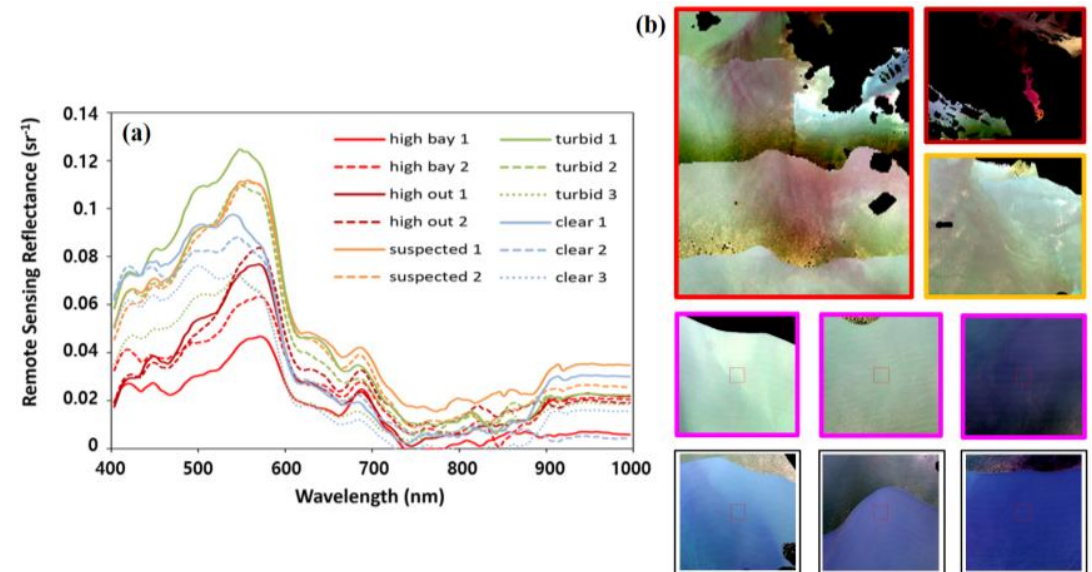

Figure 11. (a) Extracted $R_{\mathrm{rs}}$ spectrum from atmospheric-corrected airborne hyperspectral images and (b) patches from which $R_{\mathrm{rs}}$ spectrum data were extracted (Each patch's color matches with the patches in figure 9). 

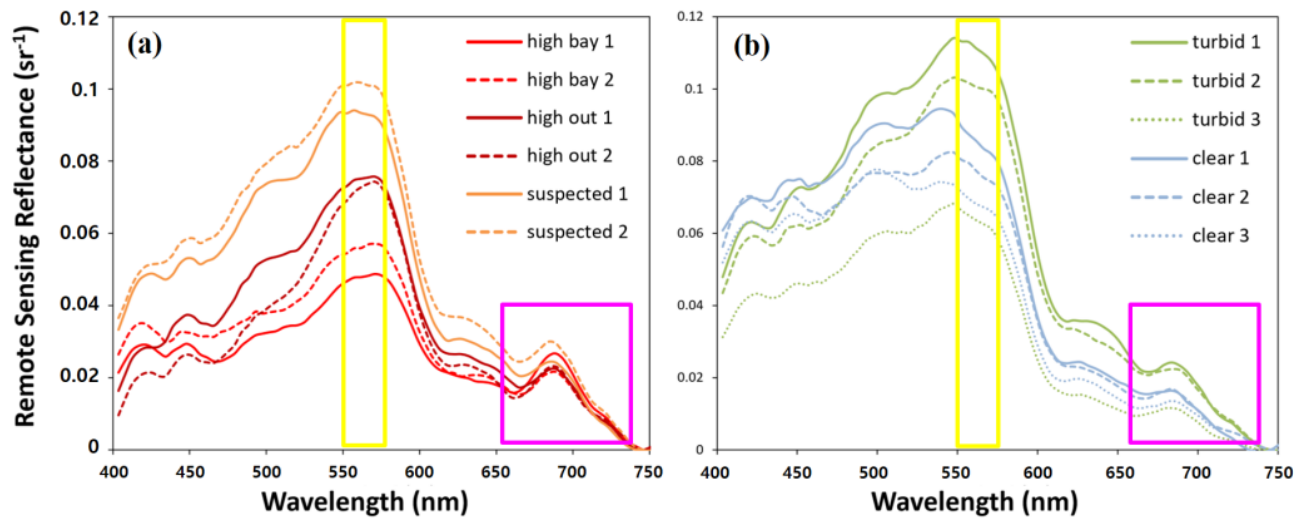

Figure 12. Comparison of the $R_{\mathrm{rs}}$ spectra from (a) red tide-suspected regions and (b) other regions (i.e., other turbid water and clear water).

the $R_{\mathrm{rs}}$ spectrum verification confirmed the effectiveness of the atmospheric correction, especially at wavelengths of $550 \sim 650$ $\mathrm{nm}$, as well as the similar shapes and values of the corrected $R_{\mathrm{rs}}$ spectra compared with the in-situ $R_{\mathrm{rs}}$ spectrum.

\subsection{Red tide detection}

We had planned to conduct the airborne hyperspectral imaging campaign during a red tide bloom. However, the bloom ended before the campaign, and therefore data collection was carried out in the absence of a bloom. Regardless, areas of potential red tide blooms were observed in the atmosphere-corrected hyperspectral imagery. In Figure 9, the areas marked with two red boxes and one yellow box represent regions with potential red tides (hereafter, red tide-suspected regions). To analyze and compare the $R_{\mathrm{rs}}$ spectra for red tide blooms with the spectra of other turbid water or clear water, the $R_{\mathrm{rs}}$ spectra were extracted from areas within the three red tide-suspected regions, three turbid water regions, and three clear water regions. The extracted
$R_{\mathrm{rs}}$ spectra are presented in Figure 11a, and Figure 11b shows the patches used for $R_{\mathrm{rs}}$ spectrum extraction. The relative position of each patch is shown in Figure 9 as a box of the same color. The $R_{\mathrm{rs}}$ values fell within a defined range without particular abnormal values, and the $R_{\mathrm{rs}}$ spectral shapes differed for each type of region. Large $R_{\mathrm{rs}}$ values within the wavelength range of $\geq 750 \mathrm{~nm}$ were excluded from the red tide analysis due to the poor performance of atmospheric correction at these wavelengths.

The $R_{\mathrm{rs}}$ spectra of red tide-suspected regions and regions with no red tides (i.e., turbid and clear water) were separated, and are displayed in Figure 12. Two notable differences were observed. First, the $R_{\mathrm{rs}}$ spectra for the red tide-suspected regions showed an increasing slope within the wavelength range of $547 \sim 575 \mathrm{~nm}$, whereas the non-red tide regions showed a decreasing slope. Second, comparatively large and prominent peaks were observed at $680 \mathrm{~nm}$, which is affected by chlorophyll fluorescence, in the $R_{\mathrm{rs}}$ spectra from red tide-suspected regions.
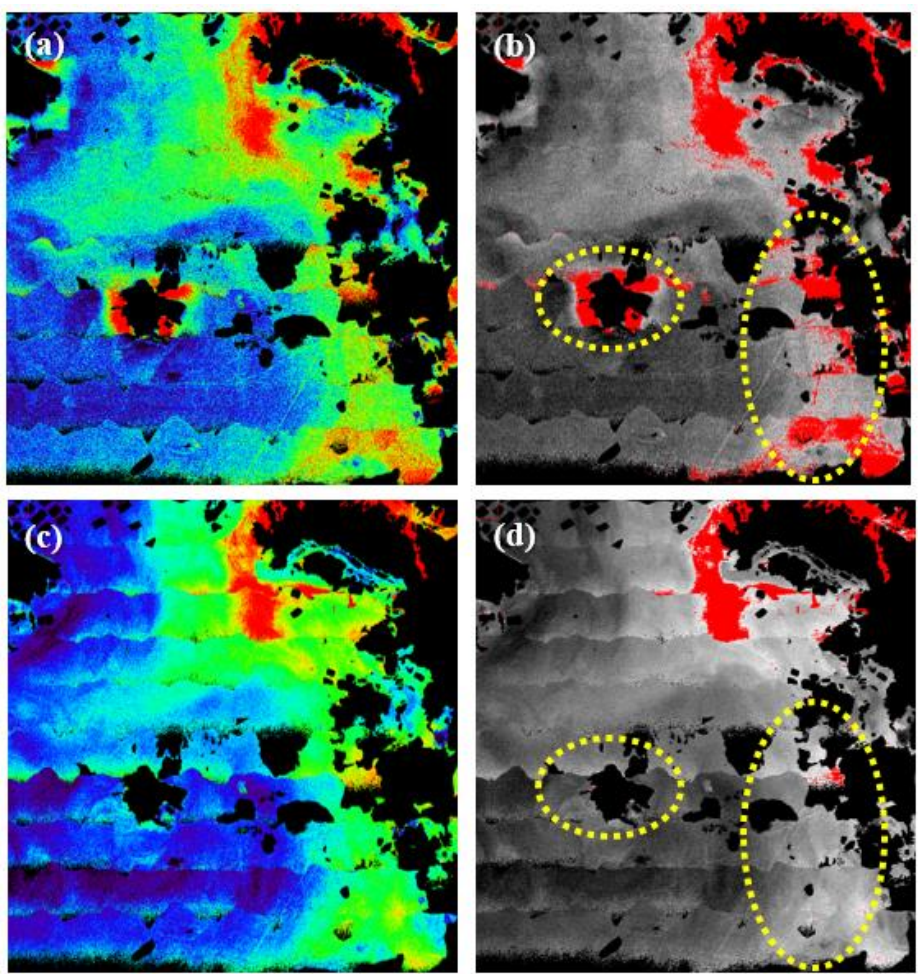

Figure 13. Red tide detection based on (a, b) fluorescence line height analysis at $680 \mathrm{~nm}$ and (c, d) ratio analysis between 547 and $575 \mathrm{~nm}$. 
To assess the potential for red tide detection using hyperspectral images, ratio analysis using $R_{\mathrm{rs}}$ values of 547 and $575 \mathrm{~nm}$ and fluorescence line height (FLH) analysis at $680 \mathrm{~nm}$ were performed. The ratio analysis was calculated by dividing the $R_{\mathrm{rs}}$ value at $547 \mathrm{~nm}$ by the $R_{\mathrm{rs}}$ value at $575 \mathrm{~nm}$. Figures $13 \mathrm{c}$ and $d$ show the ratio analysis results; red shading in Figure 13d with values greater than 1 indicates red tide-suspected regions. The FLH analysis was calculated using two base wavelengths at 657 and $731 \mathrm{~nm}$, along with one peak wavelength at $683 \mathrm{~nm}$. Figures 13a and $\mathrm{b}$ show the FLH analysis results; red shading in Figure $13 \mathrm{~b}$ indicates red tide-suspected regions. Of these two methods, the ratio analysis more effectively estimated the red tide-suspected regions, whereas the FLH analysis mis-detected some coastal turbid areas (yellow circle, Figure 13b and d).

\section{CONCLUSIONS}

In this study, an integrated field campaign was performed for the acquisition of airborne hyperspectral images combined with in situ match-up data measurements for red tide detection. For the atmospheric correction of hyperspectral images, $L_{\mathrm{a}}$ and surface reflection of $L_{\text {sky }}$ (i.e., $L_{\mathrm{r}}$ ) were eliminated from the total radiance. Additionally, land, cloud, and sun-glint areas were removed from the images. $L_{\text {a }}$ correction based on the aircraft characteristics and the relative geometry of each pixel using ATCOR was remarkably successful, when combined with $L_{\mathrm{r}}$ correction using the surface reflection model developed by Mobley (2015). In particular, $L_{\mathrm{r}}$ correction comprised a large proportion of the atmospheric correction because airborne measurements have a wide FOV. Red tide detection was analyzed using two methods, ratio analysis and the FLH technique, of which the ratio analysis more effectively predicted red tide-suspected regions.

Airborne hyperspectral imaging has many advantages in coastal red tide analysis, including the observation of large areas of coastal waters with high spatial resolution and the freedom of measuring areas of interest. In addition, spectral bands not provided by ocean color sensors could be used based on the spectral characteristics of red tides, enabling effective detection of red tide blooms. However, hyperspectral images contain large amounts of data, requiring high-end hardware and substantial time for data processing. Furthermore, precise atmospheric correction is required, considering the relative geometric position of each pixel. In this study, we found that airborne observations were heavily influenced by surface reflected radiance due to the wide FOV. At the flight planning stage, orienting the flight in the east-west direction greatly influenced surface reflection and made correction difficult. Future airborne observations should design flight plans considering the position of the sun. Moreover, based on Mobley's model, it would be appropriate to set a solarsensor azimuth between $60^{\circ}$ and $120^{\circ}$.

\section{ACKNOWLEDGEMENTS}

The authors would like to thank ASIA Aero Survey Co., Ltd. for the hyperspectral data acquisition. We also thank the members of Korea Ocean Satellite Center (KOSC) for in-situ data collection and laboratory experiments.

\section{REFERENCES}

Boss, E., D'sa, E.J., Freeman, S., Fry, E., Mueller, J.L., pegau, S., Reymolds, R.A., Roesler, C., Rotters, R., Stramski, D., Twardowski, M., \& Zaneveld, R.V., 2018. Inherent Optical Property Measurements and Protocols: Absorption Coefficient (v1.0). In N. A. R, \& A. Manni (Eds.), Ocean Optics \& Biogeochemistry Protocols for Satellite Ocean Colour Sensor Validation. Dartmouth, Canada: IOCCG.

Brando, V.E., and Dekker, A.G., 2003. Satellite hyperspectral remote sensing for estimating estuarine and coastal water quality. IEEE Trans. Geosci. Remote Sens. 41(6), 1,378-1,387.

Gao, B.C., Montes, M.J., Davis, C.O. and Goetz, A.F.H., 2009. Atmospheric correction algorithm for hyperspectral remote sensing data of land and ocean. Remote Sens. Environ. 113, S17S24.

Giardino, C., Brando, V.E., Dekker, A.G., Strombeck, N. and Candiani, G. 2007. Assessment of water quality in Lake Garda (Italy) using Hyperion. Remote Sens. Environ. 109, 183-195.

Guild1, L. S., Kudela, R. M., Hooker, S. B., Palacios, S. L. and Houskeeper, H. F., 2020. Airborne radiometry for calibration, validation, and research in oceanic, coastal, and inland waters, Front. Environ. Sci., https://doi.org/10.3389/fenvs.2020.585529

Jeffrey, S.W., \& Humphrey, G.F. 1975. New spectrophotometric equations for determining chlorophylls $\mathrm{a}, \mathrm{b}, \mathrm{c} 1$ and $\mathrm{c} 2$ in higher plants, algae and natural phytoplankton. Biochem. Physiol. Pflanz. 8, 53-59.

Karaska, M.A., Huguenin, R.L., Beacham, J.L., Wang, M.H., Jensen, J.R. and Kaufmann, R.S., 2004. AVIRIS measurements of chlorophyll, suspended minerals, dissolved organic carbon and turbidity in the Neuse River, North Carolina. Photogramm. Eng. Remote Sensing 70(1), 125-133.

Kim, D. I., Matsuyama, Y., Nagasoe, S., Yamaguchi, M., Yoon, Y. H., Oshima, Y., Imada, N. and Honjo, T., 2004. Effects of temperature, salinity and irradiance on the growth of the harmful red tide dinoflagellate Cochlodinium polykrikoides Margalef (Dinophyceae), J. Plankton Res., 26(1), 61-66.

Lee, Z.P., Carder, K.L., Chen, R.F. and Peacock, T.G., 2001. Properties of the water column and bottom derived from Airborne Bisible Infrared Imaging Spectrometer (AVIRIS) data. J. Geophys. Res. 106(C6), 11,639-11,651.

Lee, C. K., Park, T. G., Park, Y. T. and Lim, W. A. 2013. Monitoring and trends in harmful algal blooms and red tides in Korean coastal waters, with emphasis on Cochlodinium polykrikoides, Harmful Algae, 30S, S3-S14.

Mueller, J.L., Morel, A., Frouin, R., Davis, C., Arnone, R., Carder, K., Lee, Z.P., Steward, R.G., Hooker, S., Holben, B., Mobley, C.D., McLean, S., Miller, M., Knobelspiesse, K.D., Porter, J., \& Voss, K. 2003. Radiometric Measurements and Data Analysis Protocols. Maryland, US: NASA.

Mobley, C., 2015. Polarized reflectance and transmittance properties of windblown sea surfaces. Appl. Opt. 54(15), 4,8284,849 .

Mobley, C., Boss, E. and Roesler, C., 2020. Ocean optics web book. URL http://www. oceanopticsbook. info.

Olmanson, L.G., Brezonik, P.L. and Bauer, M.E., 2013. Airborne hyperspectral remote sensing to assess spatial distribution of water quality characteristics in large rivers: The Mississippi River and its tributaries in Minnesota. Remote Sens. Environ. $130,254-265$. 
Richter, R. and Schläpfer, D., 2017. Atmospheric/topographic correction for airborne imagery: ATCOR-4 User Guide, Version 7.1.1, DLR IB, 565-02.

Ryan, J. P., Fischer, A. M., Kudela, R. M., Gower, J. F. R., King, S. A., Marin III, R. and Chavez, F. P., 2009. Influences of upwelling and downwelling winds on red tide bloom dynamics in Monterey Bay, California, Cont. Shelf Res., 29, 785-795.

Shin, J., Kim, S. M., Kim, K. and Ryu, J. H., 2020. Quantification of margalefidinium polykrikoides blooms along the south coast of Korea using airborne hyperspectral imagery, Remote Sens., 12, 2463; doi:10.3390/rs12152463

Thiemann, S., and Kaufmann, H., 2002. Lake water quality monitoring using hyperspectral airborne data - a semiempirical multisensor and multitemporal approach for the Mecklenburg Lake District, Germany. Remote Sens. Environ. 81, 228-237.

Vahtmae, E., Kutser, T., Martin, G. and Kotta, J., 2006. Feasibility of hyperspectral remote sensing for mapping benthic macroalgal cover in turbid coastal waters - a Baltic Sea case study. Remote Sens. Environ. 101, 342-351. 